\title{
A novel approach towards the production of luminescent silicon nanoparticles: sputtering, gas aggregation and co-deposition with $\mathrm{H}_{2} \mathrm{O}$
}

\author{
K. von Haeften ${ }^{1, a}$, C. Binns ${ }^{1}$, A. Brewer ${ }^{1}$, O. Crisan ${ }^{1, b}$, P.B. Howes ${ }^{1}$, M.P. Lowe ${ }^{2}$, \\ C. Sibbley-Allen ${ }^{1}$, and S.C. Thornton ${ }^{1}$ \\ 1 Department of Physics \& Astronomy, University of Leicester, University Road, Leicester LE1 7RH, UK \\ 2 Department of Chemistry, University of Leicester, University Road, Leicester LE1 7RH, UK
}

Received 19 September 2008

Published online 6 February 2009 - C EDP Sciences, Società Italiana di Fisica, Springer-Verlag 2009

\begin{abstract}
Silicon clusters were produced by sputtering of a $p$-doped Si target and aggregation of the $\mathrm{Si}$ atoms in an argon gas atmosphere. The clusters were deposited in ultra high vacuum onto either (i) carbon transmission electron microscope (TEM) grids or (ii) a liquid nitrogen cooled finger on which a thick layer of ice was co-deposited during the exposure to the cluster beam. The ice layer containing the clusters was melted to form a liquid sample which showed luminescence peaking at $421 \mathrm{~nm}$ when excited at $307.5 \mathrm{~nm}$. The luminescence is attributed to electron-hole recombination in oxygen deficient defects in the $\mathrm{Si}_{-} \mathrm{SiO}_{2}$ interface region. TEM images of the nanoparticles deposited on the carbon grids show spherical particles with diameters ranging from 4 to $50 \mathrm{~nm}$, flake-like structures or nanotube-like shapes. Grids with higher deposited densities reveal clusters that are agglomerated into chains, TEM images of the dried liquid sample show a network of fibres indicating that growth into fibres is further promoted when the clusters gain mobility in the melted ice.
\end{abstract}

PACS. 36.40.Mr Spectroscopy and geometrical structure of clusters - 68.37.Lp Transmission electron microscopy (TEM) - 78.55.-m Photoluminescence, properties and materials - 78.67. Bf Nanocrystals and nanoparticles

\section{Introduction}

Optical signal transmission is expected to boost the performance of highest-integration-electronic chips in the future because with standard technology the increasingly longer interconnections will produce significant losses in speed. This problem could be overcome if, for instance, the clock-refresh pulse in storage circuits could be transmitted optically. Bulk crystalline silicon being the workhorse in microelectronics is well-known for its low luminescence efficiency due to its indirect band gap. In the past decades various forms of $\mathrm{Si}$ have been explored to overcome this limitation including porous $\mathrm{Si}[1-3]$, nanoparticle $\mathrm{Si}[4,5]$, lanthanide doped Si [6-8] and Si nanowires [9]. Molecular beam techniques have been used to produce luminescent Si nanoparticles employing a laser pyrolysis source $[10,11]$. Molecular beams have the advantage that they provide a wide range of tunability in size [12-15] as well as the means to investigate the clusters in situ in the beam $[16,17]$. Another advantage of molecular beams is that clusters can be

\footnotetext{
${ }^{a}$ e-mail: kvh6@le.ac.uk

b Now at National Inst. for Material Physics, Bukarest, Romania
}

co-doped in the vacuum by a pick-up process [18] or by codeposition with another gas onto a cold substrate $[19,20]$. In this paper we report on the molecular beam production of silicon clusters by DC magnetron sputtering and co-deposition with water onto a liquid nitrogen cooled substrate. TEM images of directly deposited clusters revealed spherical, flake and nanowire morphologies. Melted and dried Si-in-ice samples predominantly show a network of nanowire in the TEM images. The liquid samples exhibit luminescence in the blue spectral range at room temperature which is assigned to transitions at oxygen-deficient defect sites in $\mathrm{SiO}_{2}$.

\section{Experiment}

The silicon clusters in this work were produced by the magnetron gas aggregation method similar to that described by the groups of von Issendorf/Haberland $[17,21]$, Palmer [14] and Milani [15] using a DC sputter head and a gas aggregation chamber manufactured by Oxford Applied Research. The aggregation chamber was cooled with liquid nitrogen. Clusters formed in this chamber flowed through a three-stage aerodynamic lens system into a differential 


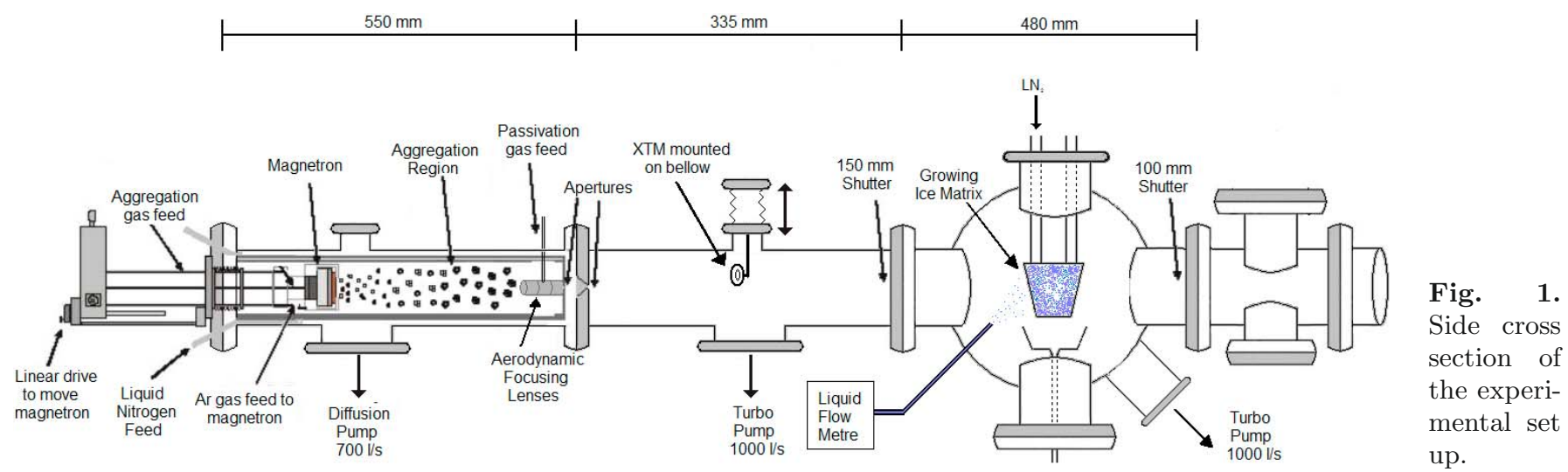

pumping stage pumped by a $700 \mathrm{~L} / \mathrm{s}$ diffusion pump and thereafter through a $3 \mathrm{~mm}$ aperture into a UHV chamber. The UHV base pressure was $\sim 1 \times 10^{-9}$ mbar but during operation the pressure rose up to $\sim 1 \times 10^{-4}$ mbar due to the high Ar gas load. The UHV chamber was equipped with a crystal thickness monitor (XTM) mounted on a linear translation stage to measure the cluster flux. A holder for TEM grids was mounted on the same translation stage. Further downstream the chamber was terminated by a gate valve that separated another UHV chamber. This second UHV chamber was equipped with a cold finger that can be filled with liquid nitrogen for cluster deposition on a cryogenic ice matrix. The water was introduced by a capillary which ended close to the cold finger and codeposited with the clusters onto the cold substrate. The water-flow through the capillary was controlled using a Bronkhorst flow controller. A cross section through the overall experimental set up is shown in Figure 1.

Silicon was sputtered from a 2 inch $p$-doped Si target (LESKER) at $80 \mathrm{~K}$ in an Ar atmosphere. The Ar was introduced through a capillary close to the discharge region and its flow was regulated by a leak valve (BOC) outside the machine where the pressure was measured by a Pirani gauge. The measured values of this pressure was between 9 and 22 mbar but we can assume that the real pressure in the sputter region was lower, possibly by a factor of two. The separation between anode and cathode was initially set to a distance of $0.3 \mathrm{~mm}$ following the manufacturer's recommendations, but for silicon a better stability in flux was achieved by using a gap of $1.8 \mathrm{~mm}$. We could only observe flux on the XTM above $40 \mathrm{~W}$ although it was possible to ignite the discharge at much lower power. When, for instance, $\mathrm{Au}$ and $\mathrm{Ag}$ were sputtered much lower power levels were needed to observe flux. Figure 2 shows that the flux dependence on the discharge power is irregular when using Si targets compared to $\mathrm{Au}$ and $\mathrm{Ag}$. The aggregation length which is the distance between the sputter head and the aerodynamic lenses was varied as well showing a maximum in flux for lengths larger than $70 \mathrm{~mm}$.

Figure 3 shows the flux dependence on the Ar pressure and similar observations as mentioned above are made when $\mathrm{Si}$ is compared with $\mathrm{Ag}$ and $\mathrm{Au}$. While the pressure dependence for the metal targets shows a clear monotonic increase with Ar pressure, a maximum around 15 mbar is

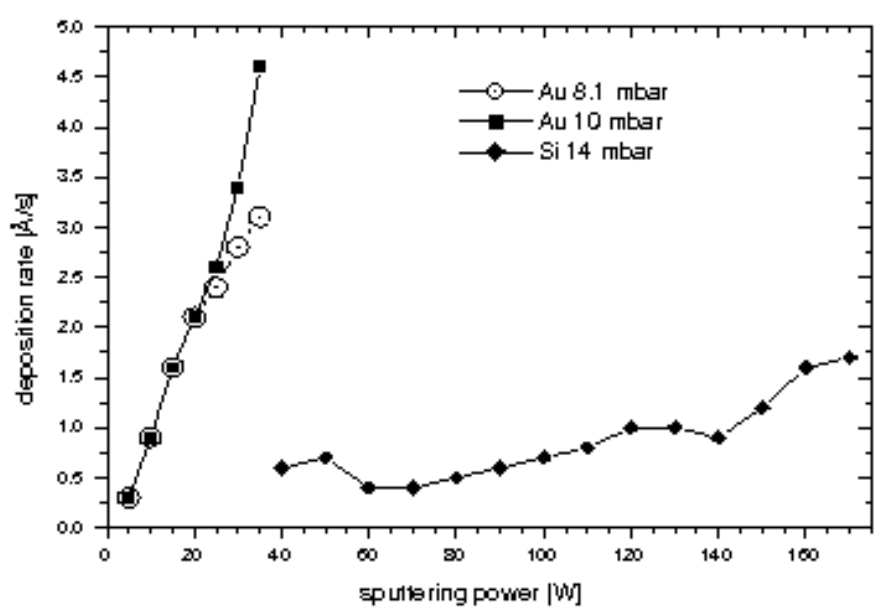

Fig. 2. Power dependence of the deposition rate at an aggregation length of $75 \mathrm{~mm}$.

observed for Si depending on the discharge power. For all conditions, the particle fluxes where lower when using $\mathrm{Si}$ compared to metal targets. In general, we found that when sputtering Si it was more difficult to achieve reliability and reproducibility. This observation was also made by other groups using similar techniques [14].

In order to vary the mean cluster size we added He into the aggregation region while reducing the Ar pressure at the same time. However, ratios with more He than Ar were impossible to establish because the discharge died down.

For further characterisation, the clusters were deposited on carbon films supported by a copper mesh (Agar) which were introduced into the molecular beam. These TEM grids were exposed to the cluster beam for $30 \mathrm{~s}$. Co-deposition with water was performed at flux rates of $0.5 \mathrm{~mL} / \mathrm{h}$. The deposition time was $30 \mathrm{~min}$. After deposition, the chamber was vented with nitrogen and the sample was melted and bottled. This procedure yielded liquid samples of typically $0.5 \mathrm{~mL}$.

The samples were investigated by TEM using a $200 \mathrm{kV}$ Jeol JEM-2100 instrument. Photoluminescence studies were performed using a FluoroMax-P Jobin-Yvon spectrometer. All spectra were corrected for the instrument response function. The spectral resolution was $2 \mathrm{~nm}$. 

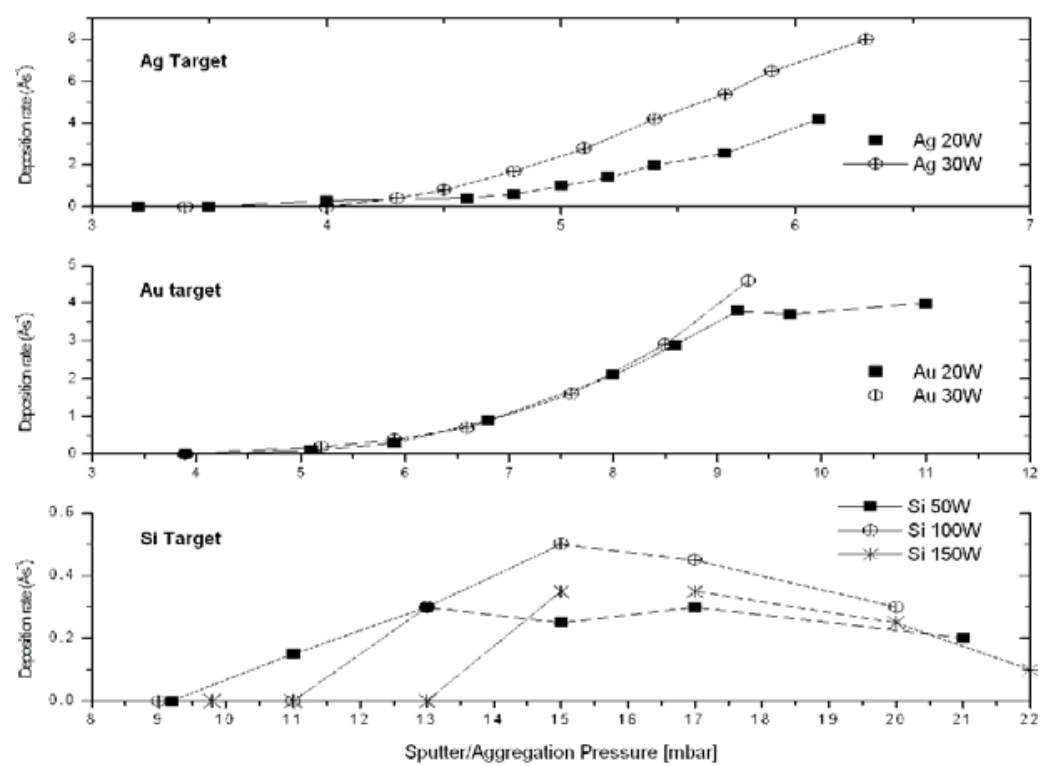

Fig. 3. Pressure dependence of the deposition rate. Aggregation length: $75 \mathrm{~mm}$.

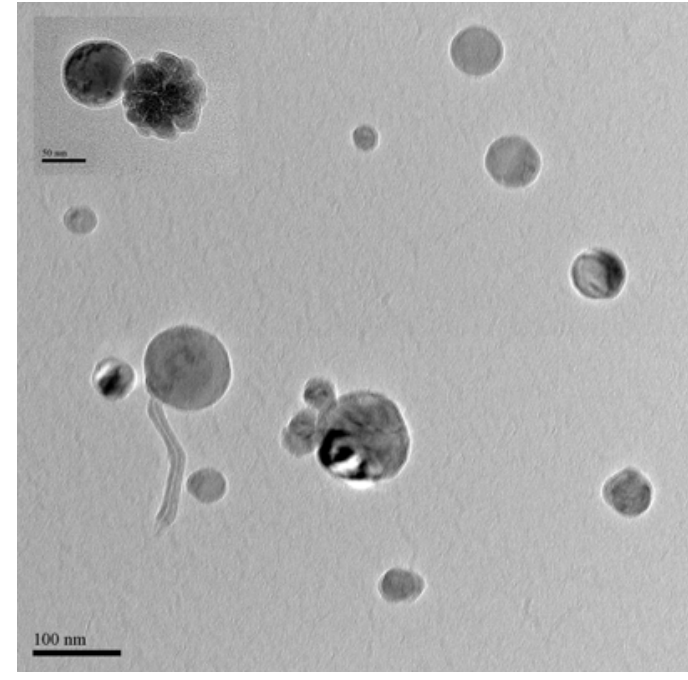

Fig. 4. TEM image showing Si spheres and wires or tubes. The inset in the upper left corner shows a flake seen in a different region of the carbon film.

\section{Results and discussion}

Inspection of TEM images of the directly deposited samples shows a variety of different shapes especially when the flux rate was showing large fluctuations. Most of the clusters are spherical but some have flake-like morphologies and a few resemble nanowires or tubes (Fig. 4).

When the deposition rate could be stabilised, the TEM image showed predominantly spherical clusters and under these conditions the size distribution of an ensemble of 250 cluster was determined from TEM images taken at lower resolution. The analyses of the TEM images from two samples produced using 12 mbar Ar and a mixture of 6 mbar Ar and 6 mbar He, respectively, revealed cluster size distribution which depended, as expected, on the Ar:He ratio with mean diameters of 15 and $5.5 \mathrm{~nm}$, re-

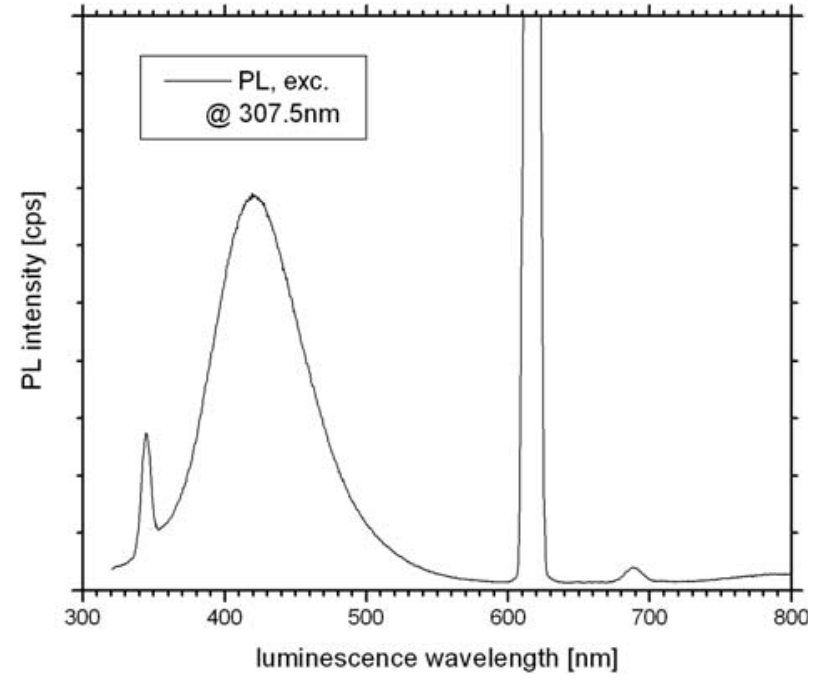

Fig. 5. Luminescence spectrum. Photo excitation energy $307.5 \mathrm{~nm}$.

spectively. The flux measured on the XTM was $7 \AA \mathrm{s}^{-1}$ for 12 mbar $\mathrm{Ar}$ and $0.8 \AA \mathrm{s}^{-1}$ for the $\mathrm{He} / \mathrm{Ar}$ mixture. In other cases we could observe a mean cluster diameter of $4 \mathrm{~nm}$ for a 1:1 ratio between $\mathrm{He}$ and $\mathrm{Ar}$ and a total pressure of 17 mbar.

Both the clusters suspended in water as well as clusters deposited on glass slides were investigated by photoluminescence spectroscopy at room temperature. The directly deposited samples showed no luminescence. In contrast all liquid samples showed room temperature luminescence. We identified a prominent band centred at $421 \mathrm{~nm}$ (Fig. 5) when excited with UV light. The maximum of this band showed only small wavelength shifts within a few nanometres with the mean cluster size. Its intensity was maximum when excited at $307.5 \mathrm{~nm}$. Figure 5 shows a representative spectrum. Apart from the above mentioned band, a few other features are visible: 


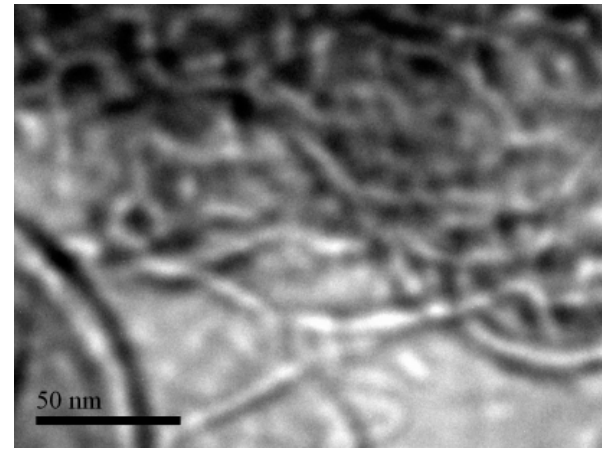

Fig. 6. TEM image of a dried sample of Si clusters codeposited onto ice.

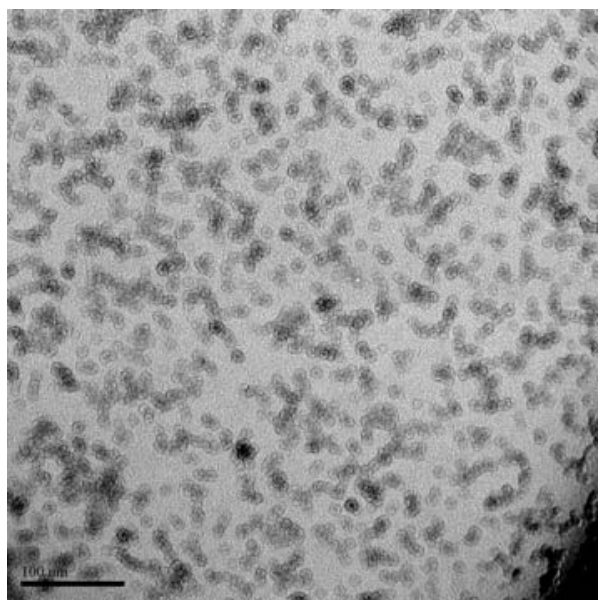

Fig. 7. Si clusters directly deposited on a TEM grid (mean diameter: $8 \mathrm{~nm}$ ). Many clusters are agglomerated to chains.

the sharp peak at $345 \mathrm{~nm}$ is the Stokes-Raman line of the water and the other sharp line at $615 \mathrm{~nm}$ is the second order of the stray light caused by the the excitation beam. A sharp peak at $690 \mathrm{~nm}$ and a weak broad band around $800 \mathrm{~nm}$ is also seen. These feature overlap with the second order spectrum but it could be possible that some intensity of the band at $800 \mathrm{~nm}$ has a similar origin as the well-known red luminescence of porous Si [3].

The fact that only small spectral shifts were observed when the cluster size is changed indicates that the origin is either due to optically active centres at the surface or due to effects that are the consequences of reactions that start after deposition in the aqueous environment so that the original size and shape of the clusters is negligible. The water provides a strongly oxidising environment causing the formation of $\mathrm{SiO}_{2}$ layers, possibly passivated with $\mathrm{H}$ or $\mathrm{OH}$. This effect alone may not be responsible for the blue luminescence because the non-luminescent, directly deposited samples were left in air where they oxidise as well. An important precondition for luminescent behaviour could be that the Si clusters gain mobility when the ice is melted and that they agglomerate into nanowires. Indeed a TEM image of a dried sample shows a network of fibres of approximately 5 to $10 \mathrm{~nm}$ in diameter. Agglomeration into wires seems to be a favourable growth process under certain conditions as can be seen from a TEM image of a dense sample of directly deposited clusters. This image shows isolated and agglomerated clusters of $8 \mathrm{~nm}$ in diameter on average (Fig. 7). Most of the agglomerated clusters are arranged in chains which must have been formed during gas phase cluster-cluster collisions in the high density region of the aerodynamic lenses. Blue luminescence from silica nanowires has been reported by several groups $[9,22]$. The luminescence around $3 \mathrm{eV}$ was related to oxygen deficient centres (ODC) such as neutral O-vacancies ( $\mathrm{Si}-\mathrm{Si}$ ) or two-fold coordinated Si-defects $(\mathrm{O}-\mathrm{Si}-\mathrm{O})$ [23]. We believe that the nanowires have a $\mathrm{Si}$ core where after light absorption excitons are created. These excitons are efficiently trapped at the ODCs at the interface to the $\mathrm{SiO}_{2}$ surface because of their mobility and their large wavefunction radius.

In conclusion, we have demonstrated a novel, molecular beam based technique for the production of luminescent $\mathrm{Si}$ nanoparticles in aqueous suspension. The origin of the luminescence has tentatively been assigned to oxygen deficient defects in the $\mathrm{SiO}_{2}-\mathrm{Si}$ interface of core shell nanowires. Due to the aqueous environment, applications in bio-sensing or medical therapy are possible.

The authors are grateful to Bernd von Issendorff for advise during the set-up of the experiment and the communication of unpublished results. Financial support by a Royal Society Research Grant (RP16G0214) and a MRC grant (86517) is kindly acknowledged.

\section{References}

1. L.T. Canham, Appl. Phys. Lett. 57, 1046 (1990)

2. A. Cullis, L. Canham, Nature 353, 335 (1991)

3. A. Cullis, L. Canham, P. Calcott, J. Appl. Phys. 82, 909 (1997)

4. T. Shimizu-Iwayama, S. Nakao, K. Saitoh, N. Itoh, J. Phys. Cond. Mat. 6, 601 (1994)

5. D. Zhang et al., Appl. Phys. Lett. 65, 2684 (1994)

6. L. Pavesi et al., Nature 408, 440 (2000)

7. L. Pavesi, J. Phys. C 15, 1169 (2003)

8. A.J. Kenyon, Semicond. Sci. Technol. 20, 65 (2005)

9. D. Yu et al., Appl. Phys. Lett. 73, 3076 (1998)

10. F. Huisken, B. Kohn, V. Paillard, Appl. Phys. Lett. 74, 3776 (1999)

11. F. Huisken, G. Ledoux, O. Guillois, C. Reynaud, Adv. Mat. 14, 1861 (2002)

12. G. Ledoux et al., Phys. Rev. B 62, 15942 (2000)

13. M. Han et al., Eur. Phys. J. D 24, 269 (2003)

14. S. Pratontep et al., Rev. Sci. Instrum. 76, 045103 (2005)

15. K. Wegner, P. Piseri, H. Tafreshi, P. Milani, J. Phys. D: Appl. Phys. 39, R439 (2006)

16. M. Ehbrecht, F. Huisken, Phys. Rev. B 59, 2975 (1999)

17. M.A. Hoffmann et al., Eur. Phys. J. D 16, 9 (2001)

18. T. Laarmann, H. Wabnitz, K. Von Haeften, T. Möller, J. Chem. Phys. 128, 014502 (2008)

19. P. Montano et al., Phys. Rev. Lett. 56, 2076 (1986)

20. M. Riedler et al., Phys. Rev. B 64, 245419 (2001)

21. H. Haberland, M. Karrais, M. Mall, Z. Phys. D 20, 413 (1991)

22. N. Shang et al., Nanotechnology 17, 3215 (2006)

23. H. Nishikawa et al., Phys. Rev. B 45, 586 (1992) 